\title{
Miocardiopatía de Takotsubo como complicación de un estado epiléptico
}

\author{
Luis A. Rodríguez de Antonio, María J. Aguilar-Amat Prior, Vicente Iváñez-Mora, Exuperio Díez-Tejedor
}

Introducción. Las complicaciones cardiológicas son la causa más frecuente de mortalidad en el estado epiléptico. La miocardiopatía de Takotsubo es una entidad descrita recientemente, que puede aparecer en numerosas emergencias médicas, entre ellas el estado epiléptico.

Caso clínico. Se presenta un caso de miocardiopatía de Takotsubo en el contexto de un estado epiléptico y se revisan casos similares descritos en la literatura científica, especialmente la semiología y etiología de las crisis epilépticas, los datos epidemiológicos de los enfermos, las alteraciones en el electrocardiograma y las complicaciones ocurridas. La paciente, una mujer de 43 años, se recuperó por completo tanto cardiológica como neurológicamente, y no tuvo recurrencias en un año de seguimiento.

Conclusión. La miocardiopatía de Takotsubo es una complicación grave y tratable que puede ocurrir en el estado epiléptico.

Palabras clave. Complicaciones. Crisis epiléptica. Epilepsia. Estado epiléptico. Miocardiopatía. Takotsubo.

\section{Introducción}

Las complicaciones cardiológicas son la principal causa de mortalidad en el estado epiléptico [1]. Aunque su mecanismo fisiopatológico no se conoce del todo, se ha propuesto que una excesiva liberación de catecolaminas podría desencadenar arritmias y producir así un fallo cardíaco [2]. Esta misma explicación etiopatogénica se ha dado para la miocardiopatía de Takotsubo.

El síndrome de Takotsubo, descrito por primera vez en Japón en 1991 [3], se caracteriza [4] por acinesia o discinesia de la porción apical y media del ventrículo izquierdo que no corresponde con un único territorio vascular en ausencia de enfermedad coronaria, demostrada mediante cateterismo cardíaco. El electrocardiograma puede mostrar elevación del segmento ST o inversión de la onda $\mathrm{T}$. Como criterios de exclusión [4], entre otros, se encuentran antecedentes recientes de hemorragia intracraneal, feocromocitoma, miocarditis, miocardiopatía hipertrófica, hipertiroidismo, pancreatitis y envenenamiento. Como desencadenantes se han relacionado factores de estrés tanto de tipo emocional como físico.

Presentamos el caso de una mujer de 43 años que en el transcurso de un estado epiléptico desarolló una miocardiopatía de Takotsubo.

\section{Caso clínico}

Mujer de 43 años con historia de epilepsia focal del lóbulo temporal izquierdo criptogénica farmacorresistente, trastorno límite de la personalidad (motivo por el cual reside en un hospital psiquiátrico), etilismo crónico, infección por el virus de la hepatitis $\mathrm{C}$ e intolerancia a múltiples fármacos antiepilépticos: fenitoína (toxicodermia), levetiracetam (insomnio), lamotrigina (diplopía). Por ello, seguía tratamiento con ácido valproico $(1.500 \mathrm{mg} / 24 \mathrm{~h})$, zonisamida (200 mg/24 h), clonacepam (1 mg/8 h), diacepam (10 mg/24 h), loracepam (1 mg/24 h) y escitalopram (20 mg/24 h).

La paciente fue ingresada en Urgencias por presentar una crisis focal motora secundariamente generalizada. Posteriormente, durante las primeras 24 horas de observación, sufrió cuatro crisis tonicoclónicas generalizadas más, que duraron entre dos y cinco minutos cada una, y que cedieron con diacepam intravenoso. Entre un episodio y otro, la paciente se encontraba somnolienta (escala de coma de Glasgow: 14/15) con una exploración neurológica, por otro lado, normal. Se trató a la paciente con ácido valproico intravenoso (dosis de carga y perfusión intravenosa) desde la primera crisis, pero tras la cuarta crisis (pasadas 24 horas desde la primera) volvió a presentar un nuevo episodio convulsivo en
Servicio de Neurología. Hospital Universitario La Paz. Madrid, España.

Correspondencia:

Dr. Luis Alberto Rodríguez de Antonio. Servicio de Neurología. Hospital Universitario La Paz. P.o de la Castellana, 261. E-28046 Madrid.

E-mail:

rodriguezdeantonio@yahoo.es

Aceptado tras revisión externa: 19.10.11.

Cómo citar este artículo: Rodríguez de Antonio LA, AguilarAmat Prior MJ, Iváñez-Mora V, Díez-Tejedor E. Miocardiopatía de Takotsubo como complicación de un estado epiléptico. Rev Neurol 2011; 53: 673-6.

C 2011 Revista de Neurología 
Figura 1. Ecocardiograma que muestra elevación del segmento ST en las derivaciones I y aVL con disminución especular del segmento ST en la cara inferior.

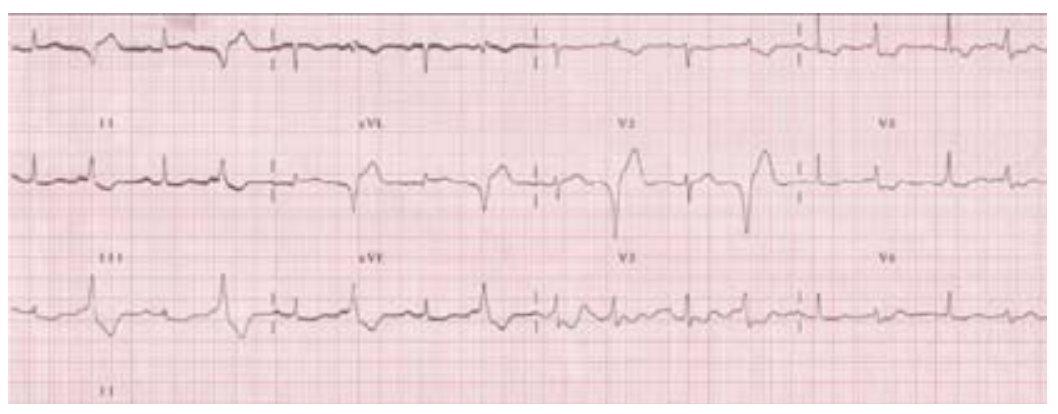

Figura 2. Cateterismo cardíaco que muestra unas arterias coronarias normales (a). Ventriculografía que revela una acinesia inferoposteroapical en sístole (b) y diástole (c).

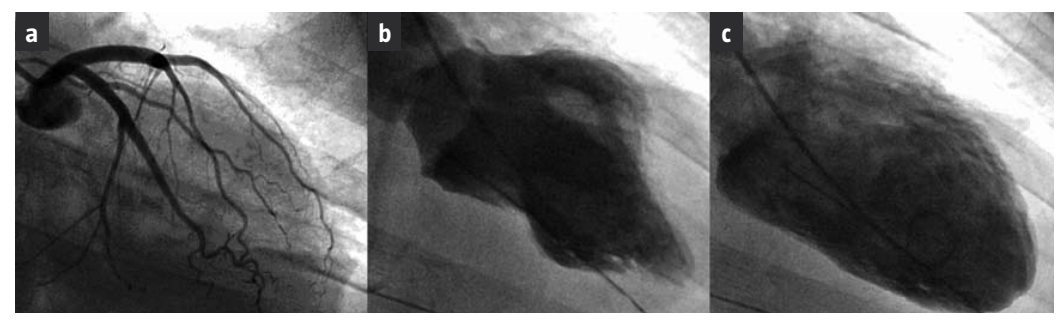

el que la frecuencia cardíaca durante la crisis descendió hasta 35 latidos por minuto. Mostró también disnea y se objetivó en la auscultación cardiopulmonar crepitantes bibasales. Ante la presencia de una bradicardia extrema, clínica compatible con shock cardiogénico y mala respuesta al tratamiento antiepiléptico, se procedió a la intubación orotraqueal y al ingreso de la paciente en la Unidad de Cuidados Intensivos.

En el electrocardiograma, realizado inmediatamente tras la intubación (Fig. 1), se objetivó una elevación del segmento ST en las derivaciones I y aVL y una disminución especular en la cara inferior. Se realizó seriación enzimática de troponina I; la primera medida fue de $0,86 \mathrm{ng} / \mathrm{mL}$ y la siguiente, seis horas después, de $44 \mathrm{ng} / \mathrm{mL}$. En el ecocardiograma transesofágico realizado en ese momento se observó acinesia de la cara lateral con fracción de eyección del ventrículo izquierdo normal. En la angiografía coronaria realizada 24 horas después no se evidenciaron alteraciones significativas, mientras que en la ventriculografía se observó una acinesia inferoposteroapical (Fig. 2). Estos hallazgos son compatibles con una miocardiopatía de Takotsubo. La paciente evolucionó favorablemente y fue trasladada a la planta de Neurología a los tres días de iniciarse el episodio. Posteriormente, no volvió a sufrir crisis epilépticas ni otras complicaciones cardiológicas durante su ingreso. Al alta se ajustó el tratamiento antiepiléptico aumentando la dosis de zonisamida hasta $200 \mathrm{mg} / 12 \mathrm{~h}$. La paciente no volvió a presentar recurrencia del cuadro tras un año de seguimiento.

\section{Discusión}

La miocardiopatía de Takotsubo -también denominada síndrome del corazón roto, miocardiopatía inducida por estrés o miocardiopatía apical [5]- es una entidad poco conocida, pero cada vez más descrita, que tiene como característica principal una disfunción intensa, pero reversible, del ventrículo izquierdo.

La miocardiopatía de Takotsubo es un síndrome recientemente descrito atribuido al exceso de catecolaminas, probablemente relacionado con una hiperactividad simpática inducida por un factor estresante de cualquier tipo. Son numerosas las enfermedades neurológicas que se han descrito relacionadas con este síndrome: hemorragia subaracnoidea [6], ictus [7], esclerosis múltiple [8], síndrome de Guillain-Barré [9], crisis miasténicas [10] y crisis epilépticas [11-19]. Del mismo modo, son numerosos los casos de síndrome de Takotsubo asociados a enfermedades psiquiátricas; de hecho, entre los enfermos descritos en la bibliografía que sufrieron crisis epilépticas coexisten varias alteraciones psiquiátricas: dependencia a opioides [16], alcoholismo [19], trastorno maníaco-depresivo [17], trastorno depresivo [13], esquizofrenia [15] y, en nuestro caso, un trastorno límite de la personalidad. También se han relacionado diversos factores emocionales (malas noticias, fiestas sorpresa, discusiones, divorcio, muertes inesperadas...) y físicos (ejercicio, neumotórax, hipoglucemia, ataque de asma, cirugías...).

Revisando los casos de estado epiléptico y miocardiopatía de Takotsubo descritos en la bibliografía (Tabla), observamos que parece ser más frecuente en el sexo femenino, en edades comprendidas entre los 50-60 años (media: 57,58 \pm 9,57 años), en epilepsias focales y agudas sintomáticas. La alteración electrocardiográfica encontrada con más frecuencia fue la elevación en el segmento ST. Las complicaciones sistémicas más habitualmente descritas en la bibliografía son la hipotensión, el edema pulmo- 
Tabla. Casos descritos en la bibliografía de miocardiopatía de Takotsubo en relación con crisis epilépticas.

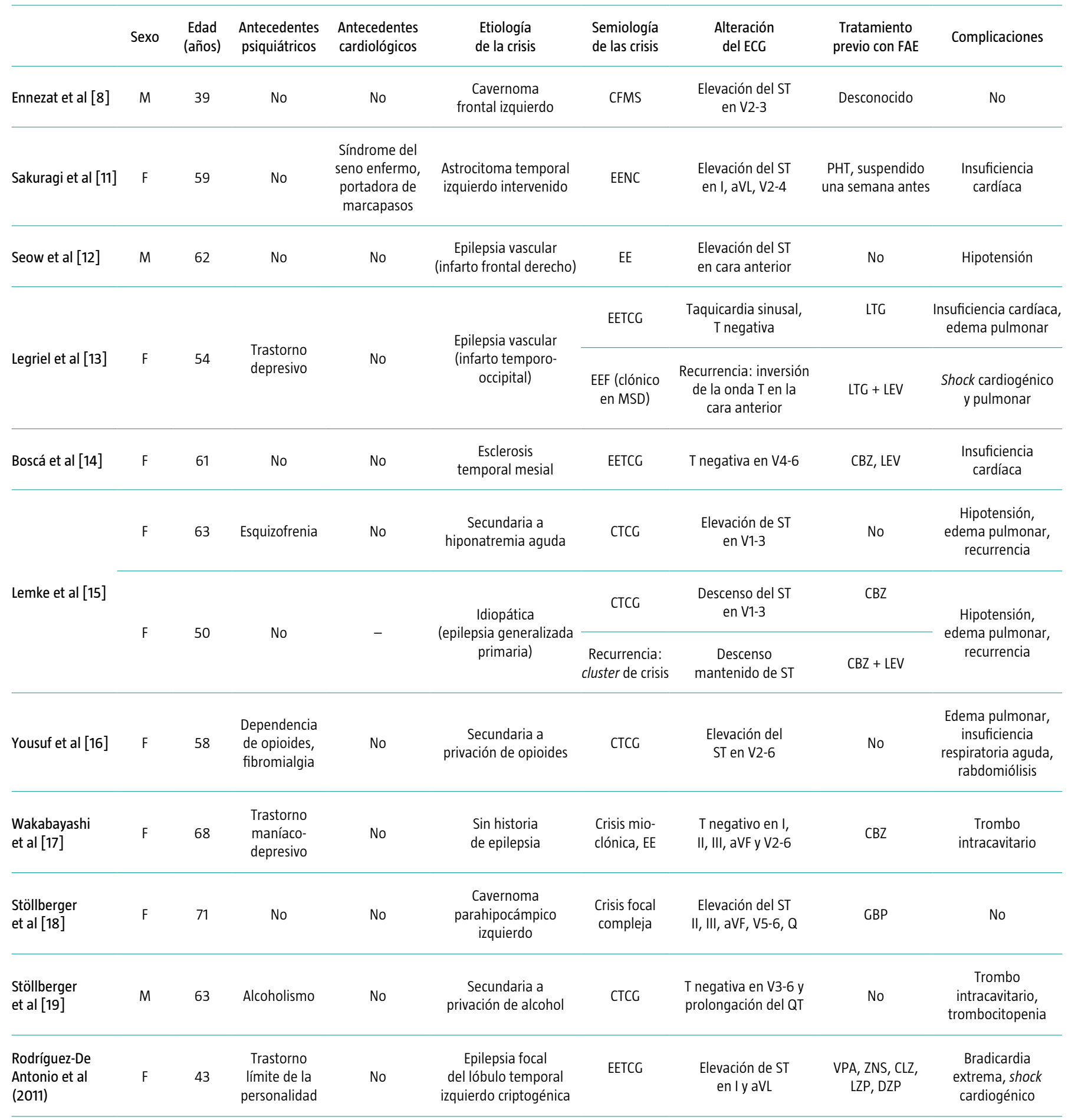

CBZ: carbamacepina; CFMS: crisis focal motora simple; CLZ: clonacepam; CTCG: crisis tonicoclónica generalizada; DZP: diacepam; ECG: electrocardiograma; EE: estado epiléptico; EEF: estado epiléptico focal; EENC: estado epiléptico no convulsivo; EETCG: estado epiléptico tonicoclónico generalizado; F: femenino; FAE: fármacos antiepilépticos; GBP: gabapentina; LEV: levetiracetam; LTG: lamotrigina; LZP: Ioracepam; M. masculino; MSD: miembro superior derecho;PHT fenitoína; VPA: ácido valproico; ZNS: zonisamida. 
nar y la insuficiencia cardíaca. Estas observaciones son similares a las de los casos de miocardiopatía de Takotsubo producidas por factores estresantes diferentes al estado epiléptico [20]. En los casos relacionados con crisis epilépticas llama la atención el elevado porcentaje de pacientes $(38,5 \%)$ que se encontraba sin tratamiento antiepiléptico previo a la crisis que desencadenó la miocardiopatía de Takotsubo. Tan sólo dos casos [13,15] presentaron recurrencia, y ésta se manifestó siempre en el primer año de seguimiento.

En conclusión, la miocardiopatía de Takotsubo es una entidad poco común, pero que debe considerarse dentro de las complicaciones cardiológicas tanto del estado epiléptico como de una crisis epiléptica, ya que, a pesar de su gravedad, si se realiza un adecuado soporte vital es reversible.

\section{Bibliografía}

1. Boggs JG, Marmarou A, Agnew JP, Morton LD, Towne AR, Waterhouse EJ, et al. Hemodynamic monitoring prior to and at the time of death in status epilepticus. Epilepsy Res 1998; 31: 199-209.

2. Boggs JG, Painter JA, DeLorenzo RJ. Analysis of electrocardiographic changes in status epilepticus. Epilepsy Res 1993; 14: 87-94.

3. Dote K, Sato H, Tateishi H, Uchuda T, Ishihara M. Myocardial stunning due to simultaneous multivessel coronary spasm: a review of 5 cases. J Cardiol 1991; 21: 203-14.

4. Bybee KA, Kara T, Prasad A, Lerman A, Barnsness GW, Wright RS, et al. Systematic review: transient left ventricular apical ballooning: a syndrome that mimics ST-segment elevation myocardial infarction. Ann Intern Med 2004; 141 858-65.

5. Kawai S, Suzuki H, Yamaguchi H, Tanaka K, Sawada H, Aizawa T, et al. Ampulla cardiomyopathy ('Takotsubo' cardiomyopathy): reversible left ventricular dysfunction with ST segment elevation. Jpn Circ J 2000; 64: 156-9.

6. Das M, Gonsalves S, Saha A, Ross S, Williams G. Acute subarachnoid haemorrhage as precipitant for Takotsubo cardiomyopathy: a case report and discussion. Int J Cardiol 2007; 115: e3-4.

7. Romano M, Achilli F, Bazzino O, Zurrú-Ganen MC Infarto cerebral como forma de presentación de un síndrome de Takotsubo. Rev Neurol 2009; 48: 558-9.

8. Ennezat PV, Pesenti-Rossi D, Aubert JM, Rachenne V, Bauchar JJ, Auffray JL, et al. Transient left ventricular basal dysfunction without coronary stenosis in acute cerebral disorders: a novel Herat syndrome (inverted Takotsubo). Echocardiography 2005; 25: 599-602.

9. Iga K, Himura Y, Izumi C, Miyamoto T, Kijima K, Gen H, et al. Reversible left ventricular dysfunction associated with Guillain-Barré syndrome: an expression of catecholamine cardiotoxicity? Jpn Circ J 1995; 59: 236-40.

10. Beydoun SR, Wang J, Levine RL, Farvid A. Emotional stress as a trigger of myasthenic crisis and concomitant Takotsubo cardiomyopathy: a case report. J Med Case Reports 2010; 4: 393.

11. Sakuragi S, Tokunaga N, Okawa K, Kakishita M, Ohe TL. A case of Takotsubo cardiomyopathy associated with epileptic seizure: reversible left ventricular wall motion abnormality and ST-segment elevation. Heart Vessels 2007; 22: 59-63.

12. Seow SC, Lee YP, Teo SG, Hong EC, Lee CH. Takotsubo cardiomyopathy associated with status epilepticus. Eur J Neurol 2008; 15: e46.

13. Legriel S, Bruneel F, Dalle L, Appere-de-Vecchi C, Georges JL, Abbosh N, et al. Recurrent Takotsubo cardiomyopathy triggered by convulsive status epilepticus. Neurocrit Care 2008; 9: 118-21.

14. Boscá ME, Valero C, Pareja AI, Bonet M, Boscá I, SánchezRoy R, et al. Takotsubo cardiomyopathy and status epilepticus: a case report. Eur J Neurol 2008; 15: e34-5.

15. Lemke DM, Hussain SI, Wolfe TJ, Torbey MA, Lynch JR, Carlin A, et al. Takotsubo cardiomyopathy associated with seizures. Neurocrit Care 2008; 9: 112-7.

16. Yousuf MA, Adjei S, Kinder B. A 58-year-old woman with ST-segment elevation, seizures, and altered mental status in the setting of opiate withdrawal. Chest 2009; 135: 1098-101.

17. Wakabayashi K, Dohi T, Daida H. Takotsubo cardiomyopathy associated with epilepsy complicated with giant thrombus. Int J Cardiol 2011; 148: e28-30

18. Stöllberger C, Huber JO, Enzelsberger B, Finsterer J. Fatal outcome of epileptic seizure-induced Takotsubo syndrome with left ventricular rupture. Eur J Neurol 2009; 16: e116-7.

19. Stöllberger C, Fischer H, Pratter A, Finsterer J. Seizureinduced Takotsubo cardiomyopathy and thrombocytopenia. Eur J Neurol 2011; 18: e68-9.

20. Fazio G, Barbaro G, Sutera L, Guttilla D, Pizzuto C, Azzarelli $S$, et al. Clinical findings of Takotsubo cardiomyopathy: results from a multicenter international study. J Cardiovasc Med (Hagerstown) 2008; 9: 239-44.

\section{Takotsubo cardiomyopathy as a complication of an epileptic status}

Introduction. Cardiological complications are the most frequent cause of mortality in the epileptic status. Takotsubo cardiomyopathy is a recently reported condition that can appear in a number of medical emergencies, including epileptic status.

Case report. We present a case of Takotsubo cardiomyopathy within the context of an epileptic status and we also review similar cases reported in the literature. Special attention is given to the semiology and aetiology of the epileptic seizures, patients' epidemiological data, the alterations noted in the electrocardiogram and the complications that occurred. The patient, a 43-year-old female, recovered completely both cardiologically and neurologically, and did not suffer any relapses during the one-year follow-up.

Conclusion. Takotsubo cardiomyopathy is a severe, treatable complication that can occur in the epileptic status.

Key words. Cardiomyopathy. Complications. Epilepsy. Epileptic seizures. Epileptic status. Takotsubo. 